\title{
Real-time pion propagation in finite-temperature QCD
}

\author{
D. T. Son ${ }^{1, \text { ⿵ }}$ and M. A. Stephanov ${ }^{2,3,}$ \\ ${ }^{1}$ Institute for Nuclear Theory, University of Washington, Seattle, Washington 98195-1550 \\ ${ }^{2}$ Department of Physics, University of Illinois, Chicago, Illinois 60607-7059 \\ ${ }^{3}$ RIKEN-BNL Research Center, Brookhaven National Laboratory, Upton, New York 11973
}

(Dated: April 2002)

\begin{abstract}
We argue that in QCD near the chiral limit, at all temperatures below the chiral phase transition, the dispersion relation of soft pions can be expressed entirely in terms of three temperaturedependent quantities: the pion screening mass, a pion decay constant, and the axial isospin susceptibility. The definitions of these quantities are given in terms of equal-time (static) correlation functions. Thus, all three quantities can be determined directly by lattice methods. The precise meaning of the Gell-Mann-Oakes-Renner relation at finite temperature is given.
\end{abstract}

PACS numbers: 11.10.Wx, 11.30.Rd

Keywords: Finite-temperature field theory, chiral symmetries

*Electronic address: son@phys.washington.edu

${ }^{\dagger}$ Electronic address: misha@uic.edu 


\section{INTRODUCTION}

Properties of hadrons at high temperatures and densities are of great interest from both experimental and theoretical perspectives. One motivation for studying temperature effects on hadrons comes from the suggestion that some features of the dilepton spectrum observed in heavy-ion collisions can be explained by the modification of masses and widths of mesons by the thermal medium [1]. Nevertheless, reliable information on the temperature modification of hadronic properties is still lacking. Lattice simulations, which rely on the imaginary-time formulation of quantum field theory, have serious difficulties with real-time quantities. ${ }^{1}$ The absence of Lorentz invariance at finite temperature implies that there is no direct relationship between real-time characteristics of hadrons (for example, the so-called "pole masses," which are supposedly the positions of poles in propagators) and quantities that can be extracted from Euclidean propagators (e.g., the "screening masses," which characterize the exponential falloff of static Euclidean correlators). Thus, as a rule, lattice measurements of correlation functions at finite temperature cannot be used to draw conclusions about real-time propagation of hadrons.

The aim of this paper is to demonstrate that pions present an exception to this rule. We shall argue that it is possible to determine the dispersion relation of soft pions (more precisely, its real part) at all temperatures below the chiral phase transition, knowing only equal-time (or static) correlation functions, which, in principle, can be determined on the lattice. It should be emphasized that we do not assume the temperature $T$ to be small compared to the chiral phase transition temperature $T_{c}$ : we must have $T<T_{c}$, but $T / T_{c}$ is allowed to be of order 1 . Our results are valid in the nontrivial regime where neither perturbative QCD nor chiral perturbation theory are reliable. Moreover, our method, with minimal modification, can be applied to any field theory with a broken symmetry, at temperatures below symmetry restoration.

That the dispersion relation of a mode can be expressed fully in terms of static correlation functions is nontrivial, but by no means unprecedented. We recall the sound waves, whose velocity is $u=(\partial p / \partial \epsilon)^{1 / 2}$, where $p$ and $\epsilon$ are the pressure and the energy density, respectively. The sound speed, while being a real-time quantity (the pole in the correlator of the energy density $T^{00}$ ), can be determined solely from thermodynamics. The relation between the speed of sound and the thermodynamic functions is an exact consequence of the existence of the hydrodynamic description. A less familiar example is the variational Feynman-Bijl formula which relates the phonon spectrum in superfluid helium to the static density-density correlation function [3]. The example most closely related to our problem, however, is that of spin waves in antiferromagnets [4]: the velocity of spin waves at any temperature below phase transition is equal to the ratio of the stiffness and the magnetic susceptibility in a direction perpendicular to magnetization. Both quantities can be defined from the static response

\footnotetext{
${ }^{1}$ Some progress, however, may have been made recently [2].
} 
of the system to external fields. The only difference between QCD and antiferromagnets is that in the former case the symmetry is $\mathrm{SU}(2)_{V} \times \mathrm{SU}(2)_{A} \simeq \mathrm{O}(4)$, which is spontaneously broken to $\mathrm{SU}(2)_{V} \simeq \mathrm{O}(3)$, while in ferromagnets $\mathrm{O}(3)$ is broken down to $\mathrm{O}(2)$ [5, 6].

The paper is constructed as follows. In Sec. II we summarize the findings of the paper. In Sec. III we use simple, but nonrigorous, arguments relying on an effective Lagrangian to understand these results. In Sec. $\mathbb{D}$ the results are derived in a more rigorous way from a set of assumptions about the real-time correlation functions, which comes from hydrodynamics. In Sec. $\mathrm{V}$ we show that our result holds for the simplest field-theoretical model of a scalar field theory with broken symmetry. In the Appendix we give a simple derivation of the known result about the dynamical critical exponent $z$, and derive the critical scaling of a diffusion coefficient.

\section{SUMMARY OF RESULTS}

We claim that, in QCD with two light flavors, at temperatures below the chiral phase transition, the real part of the dispersion relation of sufficiently soft pions is given by the following equation:

$$
\omega_{\boldsymbol{p}}^{2}=u^{2}\left(\boldsymbol{p}^{2}+m^{2}\right)
$$

In this paper the following terminology is used: $u$ is the pion velocity (although, strictly speaking, it is the pion velocity only when $m=0$ ), and $m$ is the pion screening mass (we shall show that it is the same screening mass as defined on the lattice). The energy of a pion at $\boldsymbol{p}=\mathbf{0}, m_{p}=u m$ is called the pion pole mass.

At finite temperature, the meaning of soft pions may need some clarification. We shall understand Eq. (2.1) as a statement that the correlators of operators carrying pion quantum numbers have a pole at the frequency with the real part determined by Eq. (2.1).

The pion velocity $u$ is the ratio of two statically measurable quantities, the temperaturedependent pion decay constant $f^{2}$ and the axial isospin susceptibility $\chi_{I 5}$,

$$
u^{2}=\frac{f^{2}}{\chi_{I 5}} .
$$

The axial isospin susceptibility $\chi_{I 5}$ can be defined as the second derivative of the pressure with respect to the axial isospin chemical potential (see Sec. III). Equivalently, it can be defined via the static Euclidean correlator of the axial isospin charge densities,

$$
\delta^{a b} \chi_{I 5}=\int_{0}^{1 / T} d \tau \int d \boldsymbol{x}\left\langle A_{0}^{a}(\tau, \boldsymbol{x}) A_{0}^{b}(0, \mathbf{0})\right\rangle
$$

where

$$
A_{0}^{a} \equiv \bar{\psi} \gamma^{0} \gamma^{5} \frac{\tau^{a}}{2} \psi
$$


$\psi$ is the quark field, $a, b=1,2,3, \tau^{a}$ are isospin Pauli matrices, $\operatorname{Tr} \tau^{a} \tau^{b}=2 \delta^{a b}$, and $\langle\cdots\rangle$ denotes thermal averaging, which can be taken by evaluating a Euclidean Feynman path integral with appropriate boundary conditions. The quantity $\chi_{I 5}$ has been considered previously [7].

The pion decay constant $f$ and the screening mass $m$ can be determined from the static Euclidean pion correlators at small momenta, which are predicted to have the form

$$
\int_{0}^{1 / T} d \tau \int d \boldsymbol{x} e^{-i \boldsymbol{q} \cdot \boldsymbol{x}}\left\langle\varphi^{a}(\tau, \boldsymbol{x}) \varphi^{b}(0, \mathbf{0})\right\rangle=\frac{1}{f^{2}} \frac{\delta^{a b}}{\boldsymbol{q}^{2}+m^{2}},
$$

where the scalar field $\varphi^{a}$ is defined as

$$
\varphi^{a} \equiv \frac{i \bar{\psi} \gamma^{5} \tau^{a} \psi}{\langle\bar{\psi} \psi\rangle}
$$

Equation (2.5) is supposed to be valid when $|\boldsymbol{q}| \ll m_{\sigma}$, where $m_{\sigma}$ is the (temperaturedependent) screening mass of the $\sigma$ meson.

The way $m$ enters Eq. (2.5) explains why we term it the pion screening mass. As far as $f$ is concerned, the conventional definition of the pion decay constant, $f_{\pi} p_{\mu}=\left\langle 0\left|A_{\mu}\right| \pi(p)\right\rangle$, cannot be used at finite temperature, since neither the vacuum state $|0\rangle$ nor the one-pion state $|\pi\rangle$ allows generalization in thermal media. Both $\sqrt{\chi_{I 5}}$ and $f$ approach $f_{\pi}$ as $T \rightarrow 0$, so both can be viewed as the generalization of $f_{\pi}$ to finite temperature. We, however, reserve the name "pion decay constant" for the quantity $f$ defined in Eq. (2.5). ${ }^{2}$ In contrast to the susceptibility $\chi_{I 5}, f$ cannot be defined at temperatures above critical.

We shall also show that the Gell-Mann-Oakes-Renner (GOR) relation can be generalized to finite temperature, and does in fact become two separate relations for the screening and pole masses of the pions,

$$
f^{2} m^{2}=\chi_{I 5} m_{p}^{2}=-m_{q}\langle\bar{\psi} \psi\rangle .
$$

For simplicity, in this paper we assume $m_{u}=m_{d}=m_{q}$.

Equation (2.2) is the direct analogue of a similar equation for the velocity of spin waves in a quantum antiferromagnet at temperatures below the phase transition [⿴囗十 terpart of $f$ is the stiffness (denoted as $\rho_{s}$ in Ref. [4]), while $\chi_{I 5}$ is similar to the magnetic susceptibility in a direction perpendicular to magnetization.

An important point we wish to emphasize is that the relations (2.1)-(2.7) are exact in the chiral limit, i.e., the limit when $m_{\pi}$ (vacuum pion mass) and $\boldsymbol{p}$ are infinitesimally small, at any temperature in the interval from 0 to $T_{c}$. Although the methods we use to derive them may look similar to chiral perturbation theory, unlike the chiral perturbation theory, we do not consider perturbations around $m_{\pi}=0, T=0$, treating both $m_{\pi}$ and $T$ as small

\footnotetext{
${ }^{2}$ See Sec. IVE for a discussion related to this point.
} 
parameters. Rather, we consider perturbations around $m_{\pi}=0$ at a fixed $T$, the latter not assumed to be small. Therefore, unlike in chiral perturbation theory, we are not able to calculate the temperature dependence of parameters such as $\chi, f, u$, or $\bar{\psi} \psi$. However, we are able to show that their temperature dependence must be such that relations (2.1)-(2.7) hold.

Given these equations, exact in the chiral limit, it is legitimate to ask how useful these relations can be at the physical value of the pion mass $m_{\pi}=140 \mathrm{MeV}$. To answer this question quantitatively, a (perhaps lattice) calculation of the $T$ and $m_{\pi}$ dependence is needed, which is beyond the scope of this paper. However, we shall attempt to give a semiquantitative answer to this question.

It is easier to begin with $T=0$. In this case formula (2.1) becomes trivial: the dispersion relation is exact, simply by Lorentz invariance, for any $\boldsymbol{p}$ and $m=m_{\pi}$. It is a straightforward exercise to show, using PCAC (partial conservation of axial vector current), that to leading order, i.e., $\mathcal{O}\left(m_{\pi}^{2}\right)$, the correlator in Eq. (2.3) whose value at $m_{\pi}=0$ is $\chi_{I 5}$, does not depend on $m_{\pi}$. Thus one can expect that measuring the correlator (2.3) even at the physical $m_{\pi}$ (or higher, as is typical in a lattice calculation due to the high price of simulating with light quark masses) is not significantly different from $\chi_{I 5}$. In order to extract the value of $f$ from the relation (2.5) reliably, one requires that higher mass states in the channel with pion quantum numbers do not significantly contaminate the exponential falloff of the correlator in coordinate space. Since the masses of such states are typically above $1 \mathrm{GeV}$, the exponential tail contribution from the light state of mass $m_{\pi}=140 \mathrm{MeV}$ should be easy to separate.

At nonzero $T$ the region of applicability of Eq. (2.2) is limited by terms of higher powers in $\boldsymbol{p}$. For simplicity, in the exact chiral limit $m_{\pi}=0$ the dispersion relation takes the form $\omega=|\boldsymbol{p}|-\frac{i}{2} D^{\prime} \boldsymbol{p}^{2}+\cdots$, where $D^{\prime}$ is a temperature-dependent parameter (see Sec. $\mathbb{\nabla}$ and the Appendix). The condition on momenta which is required to neglect nonlinearity in the dispersion relation is $|\boldsymbol{p}| \ll 1 / D^{\prime}$. Calculation of the diffusion constant $D^{\prime}$ is a challenging task. Taking an estimate from Ref. [8, $D^{\prime}=C T^{3} / f_{\pi}^{4}$, with a numerically rather small $C \sim 0.1$, we can conclude that even for $T \sim f_{\pi}$ at momenta of order $|\boldsymbol{p}| \sim 100 \mathrm{MeV}$ the nonlinearity in the dispersion relation is still small.

As $T=T_{c}$ the dispersion relation is essentially nonlinear, which manifests itself in the divergence of $D^{\prime}$ as $T \rightarrow T_{c}$ (see the Appendix). As $T \rightarrow T_{c}$ the maximum momentum at which the dispersion relation can be considered linear (in the chiral limit) decreases and vanishes at $T_{c}$. Although the power with which the width of the linearity window shrinks to zero can be determined (by extension of arguments given in the Appendix), the pre-exponent is unknown, and a nonperturbative calculation is, in principle, required to determine quantitatively the size of the nonlinearity in the dispersion relation at a given $T$ and $|\boldsymbol{p}|$. Such a calculation is beyond the scope of the paper.

Equations (2.1)-(2.7) were used in Ref. [9] to extract information about the critical behavior of the pion velocity and masses near the critical temperature. A brief derivation of 
Eqs. (2.1)-(2.7) was also sketched in Ref. [9]. We present a more extended version of this derivation in Sec. [III.

In Sec. IV we provide a new derivation of the relations (2.1)-(2.7) using the operator approach based on hydrodynamic equations. For this purpose we shall need expressions for $\chi_{I 5}$ and $f$ in terms of equal-time rather than static (zero frequency) correlators. These relations follow from definitions (2.3) and (2.5) in the exact chiral limit $\left(m_{\pi}=0\right)$ or when the temperature is high enough $\left(T \gg m_{\pi}\right)$ :

$$
\delta^{a b} \chi_{I 5}=\frac{1}{T} \int d \boldsymbol{x}\left\langle A_{0}^{a}(t, \boldsymbol{x}) A_{0}^{b}(t, \mathbf{0})\right\rangle .
$$

In the chiral limit, the equality of the static correlator [Eq. (2.3)] and the equal-time correlator [Eq. (2.8)] of $A_{0}^{a}$ is a consequence of the conservation of the axial isospin charge. Similarly,

$$
\frac{1}{T} \int d \boldsymbol{x} e^{-i \boldsymbol{q} \cdot \boldsymbol{x}}\left\langle\varphi^{a}(t, \boldsymbol{x}) \varphi^{b}(t, \mathbf{0})\right\rangle=\frac{1}{f^{2}} \frac{\delta^{a b}}{\boldsymbol{q}^{2}+m^{2}} .
$$

Note that, in contrast to Eqs. (2.3) and (2.5), Eqs. (2.8) and (2.9) require an additional condition $T \gg m_{\pi}$. This condition is, however, not so dramatic, if one recalls that the relavant energy scale of order $T$ is the lowest nonzero Matsubara frequency, $2 \pi T$. Thus, in practice, one requires $2 \pi T \gg m_{\pi}$, which is satisfied reasonably well for $T$ of order $T_{c} \approx 160$ $\mathrm{MeV}$ and the physical pion mass.

However, a much stronger condition is required for the applicability of the hydrodynamic description: $|\boldsymbol{p}|$ (and $m_{\pi}$ ) must be smaller than the typical collision rate $\tau^{-1} \sim T^{5} / f_{\pi}^{4}$ (according to Ref. [14). At small $T \ll f_{\pi}$ this condition is much stronger than the conditions that are required for the effective Lagrangian derivation to hold (typically $|\boldsymbol{p}|, m_{\pi} \ll m_{\sigma}-$ see Sec. [II). However, both derivations apply in the required regime infinitesimally close to the chiral limit. The fact that the hydrodynamic derivation has a smaller validity range, however, does not mean that the result is not valid outside this range. As an example, consider the case of $T=0$, where the results (2.1), (2.2) hold trivially, while hydrodynamics does not apply at all. A less trivial example is presented in Sec. $\mathrm{V}$, where explicit calculation verifies Eq. (2.2). This weak-coupling calculation, however, does not require any notion of hydrodynamics.

The advantage of the hydrodynamic approach is that it allows us to consider properly the effects of the dissipation, which are inherently beyond the Lagrangian approach. Before proceeding to the new operator derivation (Sec. IV) we review the derivation based on the effective Lagrangian approach. 


\section{EFFECTIVE LAGRANGIAN APPROACH}

\section{A. Axial isospin susceptibility and $f_{\pi}$ at $T=0$}

Since an important role in our analysis is played by the axial isospin susceptibility, we first consider this quantity at zero temperature and its relation to the pion decay constant. The quark part of the QCD Lagrangian at finite axial isospin chemical potential $\mu_{I 5}$ is given by

$$
\mathcal{L}_{\text {quark }}=i \bar{\psi} \gamma^{\mu} D_{\mu} \psi-m_{q} \bar{\psi} \psi+\mu_{I 5} \bar{\psi} \gamma_{0} \gamma_{5} \frac{\tau_{3}}{2} \psi,
$$

where $D_{\mu}$ is the color covariant derivative. This chemical potential $\mu_{I 5}$ is coupled to the axial isospin charge $A_{0}^{3}$ [cf. Eq. (2.4)], which generates the $\mathrm{SU}(2)_{A}$ part of the $\mathrm{SU}(2)_{V} \times \mathrm{SU}(2)_{A}$ chiral symmetry.

The response of the QCD vacuum to $\mu_{I 5}$ can be found from the effective chiral Lagrangian. The latter, to lowest order of momenta, masses, and chemical potential, is completely fixed by the chiral symmetries and is given by

$$
\mathcal{L}_{\text {eff }}=\frac{f_{\pi}^{2}}{4} \operatorname{Tr} \nabla^{\nu} \Sigma \nabla_{\nu} \Sigma^{\dagger}+\frac{f_{\pi}^{2} m_{\pi}^{2}}{2} \operatorname{Re} \operatorname{Tr} \Sigma
$$

where $\Sigma$ is an SU(2) matrix whose phases describe the pions, $\Sigma=e^{i \tau^{a} \pi^{a} / f_{\pi}}$, and $\nabla$ denotes the covariant derivative, which is defined as

$$
\nabla_{0} \Sigma=\partial_{0} \Sigma-\frac{i}{2} \mu_{I 5}\left(\tau_{3} \Sigma+\Sigma \tau_{3}\right), \quad \nabla_{i} \Sigma=\partial_{i} \Sigma, \quad i=1,2,3
$$

At $\mu_{I 5}=0$, the Lagrangian (3.2) is the standard chiral Lagrangian with two phenomenologically determined constants $f_{\pi}$ and $m_{\pi}$. The way $\mu_{I 5}$ enters the effective description is completely fixed by symmetries to lowest order. This can be seen by promoting the $\mathrm{SU}(2)_{A}$ symmetry to a local symmetry and treating $\mu_{I 5}$ as the time component of the $\mathrm{SU}(2)_{A}$ vector potential [10].

The Lagrangian (3.2) and its derivation is analogous to the case of the effective Lagrangian at finite (vector) isospin chemical potential $\mu_{I}$ considered in Ref. [11]. A significant difference between the cases studied here and in Ref. [11] is that the QCD vacuum breaks the $\mathrm{SU}(2)_{A}$ (axial isospin) symmetry spontaneously, but remains symmetric under the $\mathrm{SU}(2)_{V}$ (vector isospin) symmetry. It is important to note, however, that the $\mathrm{SU}(2)_{A}$ is a symmetry of the Lagrangian (at $m_{q}=0$ ), as good as the $\mathrm{SU}(2)_{V}$. In particular, the axial isospin current $A_{\mu}^{a}$ is conserved in the chiral limit. Thus, it is entirely legitimate to consider the theory at finite $\mu_{I 5}$ and use symmetry arguments to fix the $\mu_{I 5}$ dependence of the effective Lagrangian.

The vacuum energy density depends nontrivially on $\mu_{I 5}$ already for arbitrarily small $\mu_{I 5}$. (This is in contrast to the case of the isospin chemical potential $\mu_{I}$, where $\mu_{I}$ needs to be larger than a threshold equal to $m_{\pi}$ in order to change the ground state.) The isospin axial 
susceptibility, at $\mu_{I 5}=0$, is easy to determine using the effective Lagrangian (3.2):

$$
\left.\chi_{I 5} \equiv \frac{\partial^{2} \mathcal{E}_{\mathrm{vac}}}{\partial \mu_{I 5}^{2}}\right|_{\mu_{I 5}=0}=-\left.\frac{\partial^{2} \mathcal{L}_{\mathrm{eff}}}{\partial \mu_{I 5}^{2}}\right|_{\Sigma=1}=f_{\pi}^{2} .
$$

This result is of potential importance for lattice QCD calculations because, in principle, it allows one to determine $f_{\pi}$ directly by measuring the axial isospin susceptibility. To our knowledge, this has not been done on the lattice for temperatures below the chiral phase transition. The isospin susceptibility (as well as the isoscalar, i.e., baryon number susceptibility) measurement has been done using staggered fermions in [12. In the formulation used in Ref. [12], introducing the axial isospin chemical potential would require replacing $\exp (-\mu a)$ on the links (where $a$ is the lattice spacing) with $\exp \left(-\mu a \zeta_{x}\right)$, where $\zeta_{x}$ is the usual staggered factor $\zeta_{x} \equiv(-1)^{x_{1}+x_{2}+x_{3}+x_{4}}$ - the representation of $\gamma_{5}$ in the staggered fermion action.

\section{B. Pion velocity}

To obtain Eq. (2.2), we expand the previous discussion to nonzero temperature. We first presume that the dynamics of the pions is described by some effective Lagrangian $\mathcal{L}_{\text {eff }}$. Strictly speaking, this is not correct since dissipative effects cannot be included in the effective Lagrangian. We can expect to recover the correct answers if, in the infrared, the pion thermal width is negligible compared to its energy. This has been seen in explicit calculations at low $T$, where chiral perturbation theory can be used [13, 14]. We furthermore assume that this Lagrangian is local and can be expanded in powers of momenta. To lowest order, the Lagrangian is fixed by symmetries up to three coefficients, $f_{t}, f_{s}$, and $m$,

$$
\mathcal{L}_{\text {eff }}=\frac{f_{t}^{2}}{4} \operatorname{Tr} \nabla_{0} \Sigma \nabla_{0} \Sigma^{\dagger}-\frac{f_{s}^{2}}{4} \operatorname{Tr} \partial_{i} \Sigma \partial_{i} \Sigma^{\dagger}+\frac{m^{2} f_{s}^{2}}{2} \operatorname{Re} \operatorname{Tr} \Sigma
$$

Due to the lack of Lorentz invariance, $f_{t}^{2}$ and $f_{s}^{2}$ are independent parameters. The covariant derivative $\nabla_{0}$ is the same as defined in Eq. (3.3). The dispersion relation following from this Lagrangian has the form (2.1) where

$$
u=\frac{f_{s}}{f_{t}}
$$

At zero temperature $f_{t}=f_{s}=f_{\pi}$, and the pion velocity $u$ is equal to the speed of light. We now show that, at finite temperature, all three parameters $f_{t}, f_{s}$, and $m$ can be determined from equal-time (or static) correlation functions.

Repeating the same argument as in Sec. IIIA, we can show that $f_{t}$ is related to the axial isospin susceptibility,

$$
\chi_{I 5}=f_{t}^{2},
$$


but now $\chi_{I 5}$ is defined as the susceptibility at finite temperature. Note that, as a susceptibility with respect to a conserved charge, $\chi_{I 5}$ is free of ultraviolet divergences.

We now need a prescription to compute $f_{s}$ and $m$ from static correlation functions. In order to make the connection, we generalize the mass term in Eq. (3.1),

$$
\mathcal{L}_{\text {quark }}=i \bar{\psi} \gamma^{\mu} D_{\mu} \psi-\left(\bar{\psi}_{L} M \psi_{R}+\text { H.c. }\right)+\mu_{I 5} A_{0}^{3},
$$

and regard $M$ as an external field, $M=M(x)$. The Lagrangian (3.8) possesses the symmetry $\psi_{L} \rightarrow L \psi_{L}, \psi_{R} \rightarrow R \psi_{R}, M \rightarrow L M R^{\dagger}$, where $L, R \in \mathrm{SU}(2)$. If $M(x)$ is a slowly varying function of $x$, its effect can be captured in the effective chiral Lagrangian. The requirement that the effective Lagrangian preserves this symmetry fixes the form of its mass term,

$$
\mathcal{L}_{\text {eff }}=\frac{f_{t}^{2}}{4} \operatorname{Tr} \nabla_{0} \Sigma \nabla_{0} \Sigma^{\dagger}-\frac{f_{s}^{2}}{4} \operatorname{Tr} \partial_{i} \Sigma \partial_{i} \Sigma^{\dagger}-\frac{1}{2}\langle\bar{\psi} \psi\rangle \operatorname{Re} \operatorname{Tr} M^{\dagger} \Sigma .
$$

We shall limit ourselves to a particular ansatz of the external field $M(x)$ :

$$
M(x)=m_{q} e^{i \alpha^{a}(x) \tau^{a}} .
$$

The second derivative of the partition function with respect to $\alpha^{a}$ can be computed in both the microscopic theory (3.8) and in the effective theory (3.9). In the microscopic theory, we find

$$
\frac{\delta^{2} \ln \mathcal{Z}}{\delta \alpha^{a}(x) \delta \alpha^{b}(0)}=m_{q}^{2}\langle\bar{\psi} \psi\rangle^{2}\left\langle\varphi^{a}(x) \varphi^{b}(0)\right\rangle,
$$

where $\varphi^{a}$ is defined in Eq. (2.6). On the other hand, from the the effective Lagrangian (3.2) we find

$$
\frac{\delta^{2} \ln \mathcal{Z}}{\delta \alpha^{a}(x) \delta \alpha^{b}(0)}=m_{q}^{2}\langle\bar{\psi} \psi\rangle^{2}\left\langle\phi^{a}(x) \phi^{b}(0)\right\rangle, \text { where } \quad \phi^{a}(x) \equiv \operatorname{Re} \operatorname{Tr} i \tau^{a} \Sigma(x) / 2 .
$$

Comparing Eqs. (3.11) and (3.12), we see that correlation functions of $\varphi^{a}(x)$ defined in the microscopic theory and of $\phi^{a}(x)$ defined in the effective theory are equal. This equality should hold for small momenta when the effective theory is applicable.

The correlation function of $\phi^{a}(x)$, on the other hand, can be calculated by expanding the effective Lagrangian to second order in $\phi^{a}$. The Matsubara propagator of $\phi^{a}$ is

$$
\int_{0}^{1 / T} d \tau \int d \boldsymbol{x} e^{i q \cdot x}\left\langle\phi^{a}(x) \phi^{b}(0)\right\rangle=\frac{\delta^{a b}}{f_{t}^{2} q_{0}^{2}+f_{s}^{2}\left(\boldsymbol{q}^{2}+m^{2}\right)}, \quad q_{0}=2 \pi T n .
$$

This propagator, for $q_{0}=0$ and small $\boldsymbol{q}$, should be equal to the propagator of $\varphi^{a}$. This establishes Eq. (2.5), with the identification $f=f_{s}$. Together with Eqs (3.6) and (3.7), this is our result for the pion dispersion relation. Furthermore, it is natural to assume that, for small $\boldsymbol{q}$, the dynamics of $\varphi^{a}$ is slow, so at high enough temperature $\left(T \gg m_{\pi}\right)$ one can regard $\varphi^{a}(\tau, \boldsymbol{x})$ as independent of $\tau$. In this case the left hand side of Eq. (3.13) is equal to that of Eq. (2.9). 
It is instructive to write Eq. (2.9) in coordinate space,

$$
\left\langle\varphi^{a}(0, \boldsymbol{x}) \varphi^{b}(0, \mathbf{0})\right\rangle=\frac{T}{4 \pi f_{s}^{2}} \frac{e^{-m|\boldsymbol{x}|}}{|\boldsymbol{x}|} \quad\left(|\boldsymbol{x}| \gg T^{-1}, m_{\sigma}^{-1}\right) .
$$

We see that measuring, in the microscopic theory, the large-distance equal-time correlation function of the $\varphi^{a}$ defined in Eq. (2.6) we can extract the screening mass $m$ and the decay constant $f$. Combined with the determination of the susceptibility $\chi_{I 5}$ and Eq. (3.7), the dispersion relation of soft pions is now completely known.

Below we shall provide a more systematic proof of these relationships using an operator approach, with crucial inputs from the hydrodynamic theory. We shall also demonstrate the validity of the relation (2.2) in an explicit lowest order perturbative calculation in the linear sigma model.

\section{HYDRODYNAMIC (OPERATOR) APPROACH}

From a modern perspective, hydrodynamics is an effective theory operating at sufficiently large distance and time scales. (By "sufficiently large" normally we mean scales larger than the mean free path, or the relaxation time.) As such, it is the most suitable framework to discuss low-energy degrees of freedom (like pions) at finite temperatures. This modern point of view, as opposed to the view of hydrodynamics as a purely phenomenological description, has been in existence for a long time [15]. From this philosophy, it is not surprising that the same hydrodynamic theory describes systems with very different microscopic dynamics, and that systems with different symmetries (like normal fluids and superfluids) correspond to different hydrodynamic theories. Similarly to the effective Lagrangian, hydrodynamic equations can also be viewed as a particular way to satisfy Ward identities at finite temperatures [16]. In our case, hydrodynamics is nontrivial due to the chiral symmetry breaking [17]. We will derive the constraints placed by hydrodynamics on the dynamics in our problem, and show how our results on the pion dispersion relation follow from there. Our treatment is similar, to some degree, to that of Ref. [4].

\section{A. Basic assumptions of hydrodynamics}

We shall assume that, as one goes sufficiently far into the infrared, the dynamics of any interacting finite-temperature system can be described in terms of a finite number of fields, which will be called hydrodynamic variables. To be relevant in the infrared, the fluctuations of these fields (either thermal fluctuations, or those due to external sources) should relax arbitrarily slowly. This requirement eliminates most of the degrees of freedom, which typically relax during a relaxation time determined by the microscopic dynamics. However, the following fields are obvious hydrodynamic variables: 
(i) The densities of conserved quantities, including the energy density $T^{00}$, the momentum density $T^{0 i}$, and the densities of conserved global charges (i.e., the zeroth components of conserved currents). These fields cannot relax quickly because of the conservation laws. A configuration where charges fluctuate over a length scale $L$ much larger than the mean free path can relax only by diffusion, which takes place over a time proportional to $L^{2}$. The relaxation time diverges with the wavelength of the perturbation.

(ii) The phases of the condensates which break global symmetries. At zero temperature the fluctuations of such phases correspond to Goldstone bosons, whose energy can be arbitrarily small. At finite temperature below symmetry restoration, one should also expect the long-wavelength fluctuations of the condensate phases to relax slowly.

(iii) Near the critical temperatures of second-order phase transitions, the order parameters themselves (not just the phases) should be considered hydrodynamic variables [18. For example, it is believed that, in QCD with two massless flavors, the chiral phase transition is of the second order, where the $\sigma$ meson becomes degenerate with the pions. Near $T_{c}$, hence, $\sigma$ should be included in the hydrodynamic description. In contrast to the fields in the categories (i) and (ii), the rate of relaxation of order parameters is controlled by the closeness of $T$ to $T_{c}$, but not by the wavelength of perturbations. ${ }^{3}$

If one makes a further assumption that the fields listed in (i)-(iii) exhaust all slowly relaxing ones, then the set of hydrodynamic degrees of freedom is completely fixed once all symmetries of the theory and the pattern of symmetry breaking at the given temperature are known. In this paper we shall limit ourselves to temperatures far away from any second order phase transition, so order parameters are excluded from hydrodynamics. This set of hydrodynamic variables always contains the energy and momentum densities $T^{00}$ and $T^{0 i}$. For QCD below the chiral phase transition, one has, in addition, the densities of baryon, isospin, and axial isospin charges, and the phases of the chiral condensate.

After identifying the hydrodynamic degrees of freedom, one can proceed in various ways. One can ask about the the equations of motion that the hydrodynamic variables obey. For our case, it is a rather nontrivial task, because of the multitude of the fields involved. In Ref. [17], the Poisson-bracket technique is used to derive the dissipationless hydrodynamic equations. One finds a system of fully nonlinear coupled differential equations, generalizing the equations of fluid dynamics and equations of motion of the nonlinear sigma model. However, the connection of this procedure to the fundamental (microscopic) field theory has not been made, and the physical meaning of several temperature-dependent parameters appearing in the final equations (called $f_{t}, f_{s}$, and $f_{v}$ in Ref. [17]) is not at all clear.

\footnotetext{
${ }^{3}$ We shall not consider the possibility of Abelian gauge fields in the Coulomb phase (as opposed to the Higgs phase) present in magnetohydrodynamics.
} 
Alternatively, one can ask the question: what are the constraints that hydrodynamics places on the correlation functions of the hydrodynamic variables? It is clear from the discussion above that the real-time correlators of the hydrodynamic variables [i.e., $\langle\mathcal{O}(t, \boldsymbol{x}) \mathcal{O}(0, \mathbf{0})\rangle$, where $\mathcal{O}$ is the variable] are long range, i.e., have power-law (but not exponential) falloff, at least in the timelike regime $t \rightarrow \infty, \boldsymbol{x}=$ fixed. $^{4}$ It is less trivial to decide about the correlators of the operators not belonging to the set of hydrodynamic variables. To this end, the following additional assumption is made.

(iv) All local operators can be expressed as local functions of the hydrodynamic operators and their spatial derivatives, up to corrections which have short-ranged correlations that go to zero exponentially when either temporal or spatial separation goes to infinity $(t$ or $\boldsymbol{x} \rightarrow \infty)$. (These short-range parts correspond to the "noises" in the hydrodynamic theory.)

Physically, these assumptions mean that the dynamics in the infrared can be described in terms of hydrodynamic variables only, and is equivalent to the assumption of local thermodynamic equilibrium: the values of all variables are determined by specifying a few. The assumptions (i)-(iv) form the starting point of our construction of the hydrodynamic equations.

\section{B. Linearized hydrodynamics for soft pions}

To be less abstract, let us consider QCD in the chiral limit, at a finite temperature below the chiral phase transitions, and zero chemical potentials. The hydrodynamic operators are the energy density $T^{00}$, the momentum density $T^{0 i}$, the baryon density $\bar{\psi} \gamma^{0} \psi$, the densities of vector and axial isospin charges,

$$
V_{0}^{a}=\bar{\psi} \gamma^{0} \frac{\tau^{a}}{2} \psi, \quad A_{0}^{a}=\bar{\psi} \gamma^{0} \gamma^{5} \frac{\tau^{a}}{2} \psi,
$$

and the pion field, defined as Eq. (2.6),

$$
\varphi^{a}=\frac{i \bar{\psi} \gamma^{5} \tau^{a} \psi}{\langle\bar{\psi} \psi\rangle} .
$$

As we shall see below, to linear order the dynamics of $A_{0}^{a}$ and $\varphi^{a}$ is decoupled from other modes.

Let us consider the equation for $A_{0}^{a}$. Infinitesimally close to the chiral limit, we can derive from the QCD Lagrangian the familiar PCAC relation:

$$
\partial_{\mu} A^{a \mu}=m_{q}\langle\bar{\psi} \psi\rangle \varphi^{a} .
$$

\footnotetext{
4 The hydrodynamic correlators are not required to have power-law decay in the regime $t=$ fixed, $\boldsymbol{x} \rightarrow \infty$.
} 
The left hand side contains, in addition to $A_{0}^{a}$, the spatial components of the axial current $A^{a i}$, which are not hydrodynamic variables. According to the assumption (iv), we can express $A^{a i}$ as local functions of the hydrodynamic operators and their spatial derivatives, plus a short-ranged part. If we work to leading order in the power of the fields (which means the linear order), the only ones suitable are $\varphi^{a}$ and $A_{0}^{a}$ which are parity odd and isovectors. The spatial index in $A^{a i}$ forces one to have at least one spatial derivative. Thus, to leading orders in fields and derivatives, the only terms consistent with symmetries are

$$
A^{a i}=-f^{2} \partial_{i} \varphi^{a}-D \partial_{i} A_{0}^{a}-\xi^{a i},
$$

where $f^{2}$ and $D$ are coefficients depending on the temperature, and $\xi_{i}^{a}$ is the short-range ("noise") part of $A^{a i}$. (At this step, we have not yet related $f^{2}$ to the static correlation functions. We will do it later on.) Equation (4.3) now takes the form

$$
\partial_{0} A_{0}^{a}=f^{2} \nabla^{2} \varphi^{a}+m_{q}\langle\bar{\psi} \psi\rangle \varphi^{a}+D \nabla^{2} A_{0}^{a}+\partial_{i} \xi^{a i} .
$$

The parameter $D$ can be interpreted as the diffusion coefficient for $A_{0}^{a}$; however, Eq. (4.7a) is more complex than a diffusion equation. Now let us discuss the equation for $\varphi^{a}$. The time derivative of $\varphi^{a}$, not being a hydrodynamic operator, can be expanded as

$$
\partial_{0} \varphi^{a}=\frac{1}{\chi} A_{0}^{a}-\kappa_{1}^{\prime} \varphi^{a}+\kappa_{2} \nabla^{2} \varphi^{a}+\eta^{a},
$$

where $\chi, \kappa_{1}^{\prime}$, and $\kappa_{2}$ are again coefficients dependent on temperature, and $\eta$ is a short-range noise. We have kept the term with the second derivative of $\varphi^{a}$ as well as the term with no derivative. The reason for doing so is that $\kappa_{1}^{\prime}$ is suppressed by the quark masses. Indeed, in the chiral limit the state with $\left\langle\varphi^{a}\right\rangle \neq 0,\left\langle A_{0}^{a}\right\rangle=0$ can be another vacuum which stays unchanged with time. This is consistent with Eq. (4.7b) only if $\kappa_{1}^{\prime}=0$ in this limit, thus at small quark masses $\kappa_{1}^{\prime}$ is small. For this reason we keep the $\nabla^{2} \varphi^{a}$ term.

Introducing the parameter $m^{2}$ defined so that $f^{2} m^{2}=-m_{q}\langle\bar{\psi} \psi\rangle$ (which we still have to relate to static correlators), and $\kappa_{1}$ so that $\kappa_{1}^{\prime}=\kappa_{1} m^{2}$, Eqs. (4.5) and (4.6) can be written as

$$
\begin{aligned}
& \partial_{0} A_{0}^{a}=f^{2}\left(\nabla^{2}-m^{2}\right) \varphi^{a}+D \nabla^{2} A_{0}^{a}+\partial_{i} \xi^{a i}, \\
& \partial_{0} \varphi^{a}=\frac{1}{\chi} A_{0}^{a}+\left(\kappa_{2} \nabla^{2}-\kappa_{1} m^{2}\right) \varphi^{a}+\eta^{a} .
\end{aligned}
$$

Equations (4.7) are the linearized hydrodynamic equations governing the evolution of $A_{0}^{a}$ and $\varphi^{a}$. The correlation functions of $A_{0}^{a}$ and $\varphi^{a}$ can be found if one knows the correlators of the "noises" $\xi^{a i}$ and $\eta^{a}$. By construction, these fields have only short-range correlations, so, if one is interested only in the dynamics at large distance and time scales, these correlations can be replaced by delta functions. Isospin symmetry and rotational invariance require the correlators to be of the following forms:

$$
\begin{aligned}
\left\langle\xi^{a i}(x) \xi^{b j}(y)\right\rangle & =F_{\xi} \delta^{a b} \delta^{i j} \delta^{4}(x-y), \\
\left\langle\eta^{a}(x) \eta^{b}(y)\right\rangle & =F_{\eta} \delta^{a b} \delta^{4}(x-y), \\
\left\langle\xi^{a i}(x) \eta^{b}(y)\right\rangle & =0 .
\end{aligned}
$$


up to corrections proportional to derivatives of $\delta^{4}(x-y)$ which will be neglected since they are of higher order in momentum. Equations (4.7) and (4.8) completely determine the hydrodynamics of soft pions, to the linearized order.

\section{Relation to static correlators}

Our next task is to relate, as much as possible, the parameters appearing in Eqs. (4.7) and (4.8) with the equal-time correlators of $A_{0}^{a}$ and $\varphi^{a}$.

First, we multiply Eq. (4.7b) by $A^{b}$, taken at the same time moment, and integrate over space. One finds

$$
\begin{aligned}
\int d \boldsymbol{x}\left\langle\dot{\varphi}^{a}(t, \boldsymbol{x}) A_{0}^{b}(t, \mathbf{0})\right\rangle= & \int d \boldsymbol{x}\left[\frac{1}{\chi}\left\langle A_{0}^{a}(t, \boldsymbol{x}) A_{0}^{b}(t, \mathbf{0})\right\rangle-\kappa_{1} m^{2}\left\langle\varphi^{a}(t, \boldsymbol{x}) A_{0}^{b}(t, \mathbf{0})\right\rangle\right. \\
& \left.+\left\langle\eta^{a}(t, \boldsymbol{x}) A_{0}^{b}(t, \mathbf{0})\right\rangle\right] .
\end{aligned}
$$

In the right hand side, the second term is proportional to $m^{2}$, which is small in the chiral limit, and hence can be neglected. The last term in the integrand will be shown later to vanish,

$$
\left\langle\eta^{a}(t, \boldsymbol{x}) A_{0}^{b}(t, \mathbf{0})\right\rangle=0 .
$$

Equation (4.10) is trivial if understood as $\left\langle\eta^{a}(t+\varepsilon, \boldsymbol{x}) A_{0}^{b}(t, \mathbf{0})\right\rangle=0: A_{0}^{b}$ cannot be correlated with the noise $\eta$ in the future. What is somewhat less trivial (and will be checked a posteriori) is that $\left\langle\eta^{a}(t-\varepsilon, \boldsymbol{x}) A_{0}^{b}(t, \mathbf{0})\right\rangle$ also vanishes: the equal-time correlator of $\eta$ and $A_{0}$ does not depend on how the equal-time limit is taken. Therefore, only the first term survives; and by definition of $\chi_{I 5}$ it is equal to

$$
\int d \boldsymbol{x} \frac{1}{\chi}\left\langle A_{0}^{a}(t, \boldsymbol{x}) A_{0}^{b}(t, \mathbf{0})\right\rangle=\frac{T \chi_{I 5}}{\chi} .
$$

On the other hand, the left hand side of Eq. (4.9) can be computed explicitly. To this end, we write

$$
\left\langle\dot{\varphi}^{a}(\boldsymbol{x}) A_{0}^{b}(\mathbf{0})\right\rangle=i \operatorname{Tr}\left\{e^{-\beta H}\left[H, \varphi^{a}(\boldsymbol{x})\right] A_{0}^{b}(\mathbf{0})\right\} .
$$

(we drop the time variable $t$ which is an argument of all operators). We now make use of the following expansion:

$$
\left[e^{-\beta H}, \varphi^{a}\right]=-\beta e^{-\beta H}\left[H, \varphi^{a}\right]-\frac{\beta^{2}}{2} e^{-\beta H}\left[H,\left[H, \varphi^{a}\right]\right]+\cdots .
$$

The expansion parameter here is $\beta q_{0} \equiv q_{0} / T$ where $q_{0}$ is the frequency of variation of $\varphi^{a}$. Since we are dealing with the low-frequency modes in $\varphi^{a}$, we can ignore all terms beyond the first in Eq. (4.13). Equation (4.12) now becomes

$$
\left\langle\dot{\varphi}^{a}(\boldsymbol{x}) A_{0}^{b}(\mathbf{0})\right\rangle=-i T \operatorname{Tr}\left\{\left[e^{-\beta H}, \varphi^{a}(\boldsymbol{x})\right] A_{0}^{b}(\mathbf{0})\right\}=-i T\left\langle\left[\varphi^{a}(\boldsymbol{x}), A_{0}^{b}(\mathbf{0})\right]\right\rangle=T \delta^{a b} \delta^{3}(\boldsymbol{x}) .
$$


In the last transformation, we make use of the commutation relation

$$
\left[\varphi^{a}(\boldsymbol{x}), A_{0}^{b}(\mathbf{0})\right]=i \delta^{a b} \delta^{3}(\boldsymbol{x}) \frac{\bar{\psi} \psi}{\langle\bar{\psi} \psi\rangle} .
$$

Comparing to Eq. (4.11), we find

$$
\chi=\chi_{I 5}
$$

Thus, we show that $\chi$ is the axial isospin susceptibility of the system, which is a static quantity. The proof we have just presented is similar to that of the equipartition theorem in statistical mechanics.

Analogously, we can show that

$$
\int d \boldsymbol{x} e^{-i \boldsymbol{q} \cdot \boldsymbol{x}}\left\langle\dot{A}_{0}^{a}(\boldsymbol{x}) \varphi^{b}(\mathbf{0})\right\rangle=-T \delta^{a b} .
$$

On the other hand, from Eq. (4.7a)

$$
\begin{aligned}
\int d \boldsymbol{x} e^{-i \boldsymbol{q} \cdot \boldsymbol{x}}\left\langle\dot{A}_{0}^{a}(\boldsymbol{x}) \varphi^{b}(\mathbf{0})\right\rangle= & -f^{2}\left(\boldsymbol{q}^{2}+m^{2}\right) \int d \boldsymbol{x} e^{-i \boldsymbol{q} \cdot \boldsymbol{x}}\left\langle\varphi^{a}(\boldsymbol{x}) \varphi^{b}(\mathbf{0})\right\rangle \\
& -D \boldsymbol{q}^{2} \int d \boldsymbol{x} e^{-i \boldsymbol{q} \cdot \boldsymbol{x}}\left\langle A_{0}^{a}(\boldsymbol{x}) \varphi^{b}(\mathbf{0})\right\rangle,
\end{aligned}
$$

where we dropped the term proportional to

$$
\left\langle\partial_{i} \xi_{i}^{a}(t, \boldsymbol{x}) \varphi^{b}(t, \mathbf{0})\right\rangle
$$

as it will be shown to vanish in the same manner as $\left\langle\eta^{a} A_{0}^{b}\right\rangle$ in Eq. (4.9). Moreover, $D q^{2}\left\langle A_{0}^{a} \varphi^{b}\right\rangle$ can be neglected compared to $\left\langle\dot{A}_{0}^{a} \varphi^{b}\right\rangle \sim q_{0}\left\langle A_{0}^{a} \varphi^{b}\right\rangle$, since we expect the pion to have a linear dispersion relation and, for small enough momenta, $q_{0} \gg D q^{2}$. Equating Eqs. (4.17) and (4.18), we find that equal-time correlators of the pion field must have the form of a Yukawa potential,

$$
\int d \boldsymbol{x} e^{-i \boldsymbol{q} \cdot \boldsymbol{x}}\left\langle\varphi^{a}(\boldsymbol{x}) \varphi^{b}(\mathbf{0})\right\rangle=\frac{T}{f^{2}} \frac{\delta^{a b}}{\boldsymbol{q}^{2}+m^{2}} .
$$

Thus, the parameters $f^{2}$ and $m^{2}$ appearing in Eq. (4.7a) are the same ones defined in Eq. (2.9) via the equal-time correlator of $\varphi^{a}$.

The parameters $D, \kappa_{1}, \kappa_{2}, F_{\xi}$, and $F_{\eta}$ cannot be expressed individually in terms of the equal-time correlators, but some relations between them will be derived below.

\section{Hydrodynamic correlation functions}

From Eqs. (4.7) and (4.8) one can easily compute the real-time correlators of $A_{0}^{a}$ and $\varphi^{a}$ :

$$
\begin{aligned}
\int d^{4} x e^{i q \cdot x}\left\langle A_{0}^{a}(x) A_{0}^{b}(0)\right\rangle & =\delta^{a b} \frac{q_{0}^{2} \boldsymbol{q}^{2} F_{\xi}+\chi_{I 5}^{2} \omega_{\boldsymbol{q}}^{4} F_{\eta}}{\left[\left(q_{0}-\omega_{\boldsymbol{q}}\right)^{2}+\frac{1}{4} \Gamma_{\boldsymbol{q}}^{2}\right]\left[\left(q_{0}+\omega_{\boldsymbol{q}}\right)^{2}+\frac{1}{4} \Gamma_{\boldsymbol{q}}^{2}\right]}, \\
\int d^{4} x e^{i q \cdot x}\left\langle\varphi^{a}(x) \varphi^{b}(0)\right\rangle & =\delta^{a b} \frac{\chi_{I 5}^{-2} \boldsymbol{q}^{2} F_{\xi}+q_{0}^{2} F_{\eta}}{\left[\left(q_{0}-\omega_{\boldsymbol{q}}\right)^{2}+\frac{1}{4} \Gamma_{\boldsymbol{q}}^{2}\right]\left[\left(q_{0}+\omega_{\boldsymbol{q}}\right)^{2}+\frac{1}{4} \Gamma_{\boldsymbol{q}}^{2}\right]}
\end{aligned}
$$


where

$$
\begin{aligned}
\omega_{\boldsymbol{q}}^{2} & =\frac{f^{2}}{\chi_{I 5}}\left(\boldsymbol{q}^{2}+m^{2}\right), \\
\Gamma_{\boldsymbol{q}} & =\kappa_{1} m^{2}+\left(D+\kappa_{2}\right) \boldsymbol{q}^{2} .
\end{aligned}
$$

The correlation functions (4.21) peak around $q_{0} \approx \pm \omega_{\boldsymbol{q}}$, and the width of the peaks $\Gamma_{\boldsymbol{q}}$ is much smaller than $\omega_{\boldsymbol{q}}$ at small $\boldsymbol{q}$. The correlators have poles corresponding to the pion collective excitations with the dispersion relation $q_{0}=\omega_{\boldsymbol{q}}-\frac{i}{2} \Gamma_{\boldsymbol{q}}$. We note, moreover, that all parameters entering the real part of the dispersion relation (4.22a) can be determined from the static correlation functions.

It is now possible to explicitly check that the noise-field correlators appearing in Eqs. (4.10) and (4.19) vanish. Indeed, by using Eqs. (4.7) and (4.8) we find

$$
\begin{aligned}
\left\langle\eta(t, \boldsymbol{x}) A_{0}\left(t^{\prime}, \mathbf{0}\right)\right\rangle & =\delta^{a b} \int \frac{d^{4} q}{(2 \pi)^{4}} e^{-i q_{0}\left(t-t^{\prime}\right)+i \boldsymbol{q} \cdot \boldsymbol{x}} \frac{\chi_{I 5} \omega_{\boldsymbol{q}}^{2} F_{\eta}}{q_{0}^{2}-\omega_{\boldsymbol{q}}^{2}+i q_{0} \Gamma_{\boldsymbol{q}}} \\
\left\langle\partial_{i} \xi_{i}(t, \boldsymbol{x}) \varphi\left(t^{\prime}, \mathbf{0}\right)\right\rangle & =-\delta^{a b} \int \frac{d^{4} q}{(2 \pi)^{4}} e^{-i q_{0}\left(t-t^{\prime}\right)+i \boldsymbol{q} \cdot \boldsymbol{x}} \frac{\chi_{I 5}^{-1} \boldsymbol{q}^{2} F_{\xi}}{q_{0}^{2}-\omega_{\boldsymbol{q}}^{2}+i q_{0} \Gamma_{\boldsymbol{q}}} .
\end{aligned}
$$

The integrands in Eqs. (4.23) have two poles, both located in the upper half plane: $q_{0}=$ $\pm \omega_{\boldsymbol{q}}+\frac{i}{2} \Gamma_{q}$. When $t>t^{\prime}$, when taking integrals over $q_{0}$, one can close the contour in the lower half plane, so the integrals are obviously zero, which corresponds to our previous remark that the fields cannot correlate with the noises in the future. If one takes $t \rightarrow t^{\prime}$ from below, the integrals are also zero since they are equal to the sums of the residues of the integrands (which are zero because these functions behave as $q_{0}^{-2}$ at large $q_{0}$.) Therefore, we have checked that the equal-time noise-field correlators (4.10) and (4.19) indeed vanish.

One can find the relations between the amplitude of the noise correlators, $F_{\xi}$ and $F_{\eta}$, and the parameters characterizing the damping in Eq. (4.22b). One integrates Eqs. (4.21) over $q_{0}$ and obtains the equal-time correlation functions

$$
\begin{aligned}
\int d \boldsymbol{x} e^{-i \boldsymbol{q} \cdot \boldsymbol{x}}\left\langle A_{0}^{a}(t, \boldsymbol{x}) A_{0}^{b}(t, \mathbf{0})\right\rangle & =\delta^{a b} \frac{\boldsymbol{q}^{2} F_{\xi}+\chi_{I 5}^{2} \omega_{\boldsymbol{q}}^{2} F_{\eta}}{2 \Gamma_{\boldsymbol{q}}}, \\
\int d \boldsymbol{x} e^{-i \boldsymbol{q} \cdot \boldsymbol{x}}\left\langle\varphi^{a}(t, \boldsymbol{x}) \varphi^{b}(t, \mathbf{0})\right\rangle & =\delta^{a b} \frac{\boldsymbol{q}^{2} F_{\xi}+\chi_{I 5}^{2} \omega_{\boldsymbol{q}}^{2} F_{\eta}}{2 \chi_{I 5}^{2} \omega_{\boldsymbol{q}}^{2} \Gamma_{\boldsymbol{q}}} .
\end{aligned}
$$

Comparing these correlators with Eqs. (2.8) and (2.9), taking into account Eqs. (4.22), one finds

$$
\begin{aligned}
& F_{\xi}=2 T \chi_{I 5}\left(D+\kappa_{2}-\kappa_{1}\right), \\
& F_{\eta}=\frac{2 T \kappa_{1}}{f^{2}}
\end{aligned}
$$

Our results for the hydrodynamic correlations functions are given by Eqs. (4.21) and (4.25). For completeness, we give here also the result for the cross correlator of $\varphi^{a}$ and $A_{0}^{a}$ :

$$
\int d^{4} x e^{i q \cdot x}\left\langle\varphi^{a}(x) A_{0}^{b}(0)\right\rangle=\frac{2 i T q_{0} \Gamma_{\boldsymbol{q}}}{\left[\left(q_{0}-\omega_{\boldsymbol{q}}\right)^{2}+\frac{1}{4} \Gamma_{\boldsymbol{q}}^{2}\right]\left[\left(q_{0}+\omega_{\boldsymbol{q}}\right)^{2}+\frac{1}{4} \Gamma_{\boldsymbol{q}}^{2}\right]} .
$$


It is instructive to compare the correlator of the axial isospin charge density $A_{0}^{a}$ [Eq. (4.21a)] with that of the vector isospin charge density $V_{0}^{a}$. The dynamics of the latter is completely diffusive and is given by the equations

$$
\begin{aligned}
\partial_{0} V_{0}^{a}-D_{I} \nabla^{2} V_{0}^{a} & =\partial_{i} \zeta^{a i}, \\
\left\langle\zeta^{a i}(x) \zeta^{b j}(0)\right\rangle & =2 T D_{I} \chi_{I} \delta^{a b} \delta^{i j} \delta^{4}(x),
\end{aligned}
$$

where $D_{I}$ is the diffusion constant for the isospin charge, and $\chi_{I}$ is the (vector) isospin susceptibility. The correlator of $V_{0}^{a}$ is

$$
\int d^{4} x e^{i q \cdot x}\left\langle V_{0}^{a}(x) V_{0}^{b}(0)\right\rangle=\frac{2 T D_{I} \chi_{I} \boldsymbol{q}^{2}}{q_{0}^{2}+D_{I}^{2} \boldsymbol{q}^{4}} .
$$

The pole of the correlator is located at purely imaginary frequencies $q_{0}= \pm i D_{I} \boldsymbol{q}^{2}$, as it should be for a purely diffusive mode.

Using Eqs. (4.28) and (4.24) we can understand the limitations of the Lagrangian approach and the role of the dissipative processes. For example, one could try to apply the method of Sec. III to determine the vector isospin susceptibility $\chi_{I}$, introducing an isospin chemical potential $\mu_{I}$. At zero temperature such a method was used in Ref. [11. As expected, the isospin susceptibility $\chi_{I}$ vanishes at $T=0$ since it takes finite energy to excite isospin degrees of freedom (pions) and change the isospin density. However, at nonzero temperature, the naive application of the effective Lagrangian method would predict that $\chi_{I}=0$ also. $^{5}$ On the other hand, we should expect $\chi_{I} \neq 0$ at finite temperature, even at very small $T$, because of the presence of isospin-carrying pions in the thermal medium. Looking at the correlator (4.28), one sees that the equal-time correlator defining $\chi_{I}$, being the integral of Eq. (4.28) over $q_{0}$ in the limit $\boldsymbol{q} \rightarrow 0$, receives the main contribution from very slow (diffusive) modes: $q_{0} \sim D_{I} \boldsymbol{q}^{2}$. However, the effective Lagrangian (3.5) only describes faster (propagating) modes of the $\varphi$ field with $q_{0}=\omega_{\boldsymbol{q}} \gg D_{I} \boldsymbol{q}^{2}$. The slow diffusive modes which contribute to $\chi_{I}$ are not present in the Lagrangian (3.5). For the axial isospin susceptibility $\chi_{I 5}$, the situation is different, for the integral of Eq. (4.28) over frequencies in the limit $\boldsymbol{q} \rightarrow 0$ is concentrated entirely near values $q_{0}= \pm \omega_{\boldsymbol{q}}$. Thus, unlike $\chi_{I}$, the value of $\chi_{I 5}$ at finite $T$ can be found correctly using the effective Lagrangian approach.

\section{E. Comparison to previous results}

As a by-product of our analysis, we obtain a generalization of the Gell-Mann-OakesRenner relation to finite temperature:

$$
f^{2} m^{2}=-m_{q}\langle\bar{\psi} \psi\rangle .
$$

${ }^{5}$ We thank T. Schäfer for pointing this out to us. 
This equation has the same form as at zero temperature. At finite temperature, $m$ should be understood as the pion screening mass, and the exact meaning of the temperature-dependent "pion decay constant" $f^{2}$ is given by Eq. (4.20). One can also write the GOR relation in an alternative form,

$$
\chi_{I 5} m_{p}^{2}=-m_{q}\langle\bar{\psi} \psi\rangle,
$$

where $m_{p}=u m$ is the "pole mass" of pions.

That the velocity of pions at finite temperature is different from the velocity of light has been seen in the second order of chiral perturbation theory (i.e., in order $T^{4} / f_{\pi}^{4}$ ) [19, 20]. The authors of Ref. [19] also introduced two pion decay constants $f^{t}$ and $f^{s}$, which correspond to our $\sqrt{\chi_{I 5}}$ and $f$, and checked the validity of the GOR relation (4.30). However, these constants were defined only in chiral perturbation theory, and only at small $T$; there has not been any attempt to give a precise definition of the constants $f^{t}$ and $f^{s}$ at temperatures comparable to $T_{c}$. We have, in contrast, given a precise meaning to the constants $\chi_{I 5}$ and $f$ in terms of equal-time correlation functions that can be measured on the lattice. The GOR relation now contains only well-defined quantities. Although Eq. (4.30) cannot be checked on the lattice, since it contains the pion pole mass, the version (4.29) can be verified numerically since all quantities entering it are statically measurable.

We also note that Eqs. (4.7), without the noise and dissipation terms, can be obtained by linearizing the hydrodynamic equations obtained in Ref. [17] by the Poisson-bracket technique. One can then identify the parameters $f_{t}$ and $f_{s}$ in Ref. [17] as $f_{t}^{2}=\chi_{I 5}, f_{s}^{2}=f^{2}$ (the parameter $f_{v}^{2}$ of Ref. [17]) is equal to the vector isospin susceptibility). We did not try to reproduce the full nonlinear hydrodynamic equation of Ref. [17] in the present approach.

\section{AN EXPLICIT EXAMPLE: A LINEAR SIGMA MODEL}

It is instructive to explicitly verify our relation (2.2) between the velocity of the Goldstone bosons, the axial isospin susceptibility, and the temperature-dependent decay constant in a model where weak-coupling calculations are possible. The simplest theory with spontaneous breaking of a continuous symmetry is the linear sigma model. We shall show that Eq. (2.2) indeed holds in this model to leading order of perturbation theory

It is important to emphasize that the sigma model considered in this section is only meant to serve as an example of a theory where we can explicitly check our results by perturbative calculations. These models do not describe QCD at $T$ of order $T_{c}-$ a theory with no apparent small parameter. We claim, however, that the connection between the dispersion relation of the pions and static correlation functions is model independent, and can be derived from only a few general assumptions stated in Sec. IV.

We start with the following Lagrangian

$$
\mathcal{L}=\frac{1}{2} \partial^{\mu} \phi^{a} \partial_{\mu} \phi^{a}+\frac{\mu^{2}}{2} \phi^{a} \phi^{a}-\frac{\lambda}{4}\left(\phi^{a} \phi^{a}\right)^{2},
$$


where $a=1,2, \ldots, N$. We assume $\lambda \ll 1$, so perturbation theory can be applied. When $\mu^{2}>0$, the $O(N)$ symmetry is spontaneously broken at zero temperature. We choose the vacuum to align in the $\phi^{N}$ direction. At zero temperature, and at tree level, the vacuum expectation value of $\phi^{N}$ is

$$
\left\langle\phi^{N}\right\rangle \equiv v_{0}=\frac{\mu^{2}}{\lambda}
$$

At finite temperature the expectation value of $\phi^{N}$ is different from $v_{0}$. Denoting it as $v$ and replacing $\phi^{N}=v+\sigma$ in the Lagrangian (5.1), we obtain

$$
\begin{aligned}
\mathcal{L}= & \frac{1}{2}\left(\partial_{\mu} \sigma\right)^{2}+\frac{1}{2}\left(\partial_{\mu} \boldsymbol{\pi}\right)^{2}-\left(\lambda v^{2}-\mu^{2}\right) v \sigma-\frac{1}{2}\left(3 \lambda v^{2}-\mu^{2}\right) \sigma^{2}-\frac{1}{2}\left(\lambda v^{2}-\mu^{2}\right) \boldsymbol{\pi}^{2} \\
& -\lambda v \sigma^{3}-\lambda v \sigma \boldsymbol{\pi}^{2}-\frac{\lambda}{4} \sigma^{4}-\frac{\lambda}{4}\left(\boldsymbol{\pi}^{2}\right)^{2}-\frac{\lambda}{2} \sigma^{2} \boldsymbol{\pi}^{2},
\end{aligned}
$$

where $\boldsymbol{\pi}=\left(\phi^{1}, \phi^{2}, \ldots, \phi^{N-1}\right)$. From Eq. (5.3) the Feynman rules can be easily written down.

The value of $v$ is determined by the condition that $\langle\sigma\rangle=0$. For computation, we will use the Matsubara (Euclidean) formalism, in which this condition reads, to one-loop order,

$$
\left(\lambda v^{2}-\mu^{2}\right) v+\lambda v T \sum_{p_{0}} \int \frac{d \boldsymbol{p}}{(2 \pi)^{3}}\left(\frac{3}{P^{2}+m_{\sigma}^{2}}+\frac{N-1}{P^{2}}\right)=0,
$$

In this Section, $P^{2}=p_{0}^{2}+p^{2}, p=|\boldsymbol{p}|$. The sum-integral in Eq. (5.4) is ultraviolet divergent. This temperature-independent divergence can be absorbed into the redefinition of $\mu^{2}$. The temperature dependence of $v$ comes from the thermal part of the Lagrangian. We shall be interested in temperatures of order $v_{0}$, so $T \gg m_{\sigma}$. In this case,

$$
\lambda v^{2}-\mu^{2}+\frac{N+2}{12} \lambda T^{2}=0,
$$

or

$$
v^{2}(T)=v_{0}^{2}\left(1-\frac{T^{2}}{T_{c}^{2}}\right) \quad \text { with } \quad T_{c}^{2}=\frac{12}{N+2} \frac{\mu^{2}}{\lambda} .
$$

Equation (5.6) is valid everywhere except for a narrow Ginzburg region near $T_{c}$.

The masses of $\sigma$ and $\pi$ are computed, e.g., in Ref. [21]. At the one-loop level $m_{\sigma}$ receives contributions from four diagrams
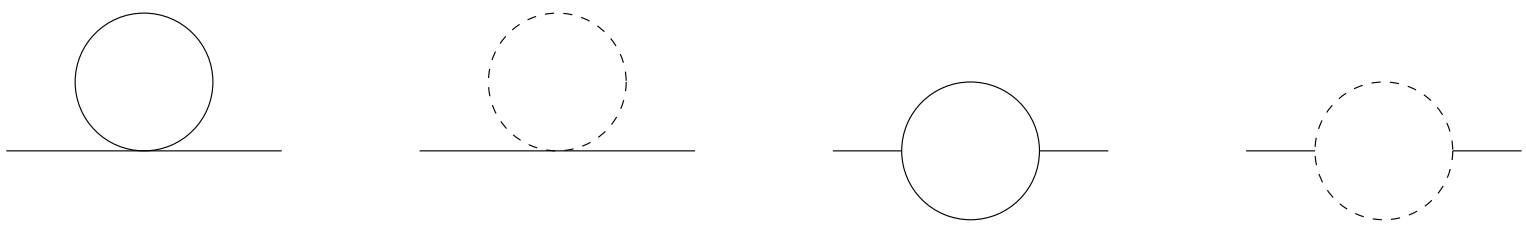

(where $\sigma$ propagators are denoted by solid lines, and $\pi$ propagators are drawn as dashed lines). The last two bubble diagrams are negligible in the finite-temperature regime we consider, so

$$
m_{\sigma}^{2}=3 \lambda v^{2}-\mu^{2}+\lambda v T \sum_{p_{0}} \int \frac{d \boldsymbol{p}}{(2 \pi)^{3}}\left(\frac{3}{P^{2}+m_{\sigma}^{2}}+\frac{N-1}{P^{2}}\right)=2 \lambda v^{2},
$$


and thus decreases with temperature. The Goldstone boson receives corrections to its mass from three one-loop graphs,

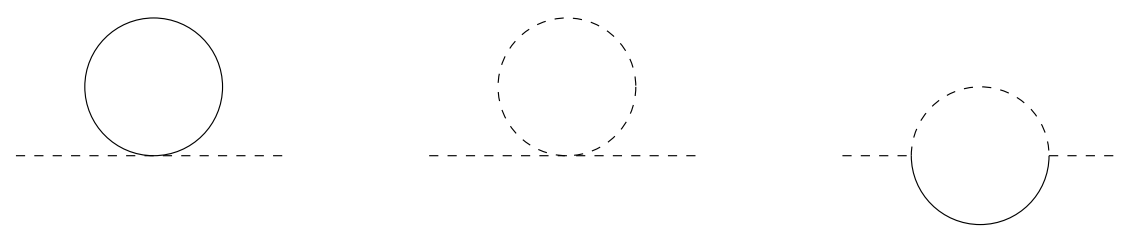

and remains massless since

$$
\begin{aligned}
m_{\pi}^{2}= & \lambda v^{2} \mu^{2}-\mu^{2}+\lambda v T \sum_{p_{0}} \int \frac{d \boldsymbol{p}}{(2 \pi)^{3}}\left(\frac{1}{P^{2}+m_{\sigma}^{2}}+\frac{N-1}{P^{2}}\right) \\
& -4 \lambda^{2} v^{2} T \sum_{p_{0}} \int \frac{d \boldsymbol{p}}{(2 \pi)^{3}} \frac{1}{P^{2}\left(P^{2}+m_{\sigma}^{2}\right)}=0 .
\end{aligned}
$$

To find the dispersion relation of the pion near $q=0$, one has to expand the pion selfenergy $\Sigma(q)$ in powers of $q_{0}$ and $\boldsymbol{q}$. The only one-loop diagram that depends on $q$ is the bubble diagram, which can be evaluated either by using the Schwinger-Keldysh real-time formalism, or by doing calculations in the Matsubara formalism and then performing an analytic continuation from imaginary to real $q_{0}$. By either method one finds

$$
\begin{aligned}
\Sigma(q)= & -4 \lambda^{2} v^{2} \int \frac{d \boldsymbol{p}}{(2 \pi)^{3} 2 \omega_{\boldsymbol{p}+\boldsymbol{q}} 2 \Omega_{\boldsymbol{p}}}\left\{\left[f\left(\omega_{\boldsymbol{p}+\boldsymbol{q}}\right)+f\left(\Omega_{\boldsymbol{p}}\right)+1\right]\left(\frac{1}{\Omega_{\boldsymbol{p}}+\omega_{\boldsymbol{p}+\boldsymbol{q}}-q_{0}}+\frac{1}{\Omega_{\boldsymbol{p}}+\omega_{\boldsymbol{p}+\boldsymbol{q}}+q_{0}}\right)\right. \\
& \left.+\left[f\left(\omega_{\boldsymbol{p}+\boldsymbol{q}}\right)-f\left(\Omega_{\boldsymbol{p}}\right)\right]\left(\frac{1}{\Omega_{\boldsymbol{p}}-\omega_{\boldsymbol{p}+\boldsymbol{q}}-q_{0}}+\frac{1}{\Omega_{\boldsymbol{p}}-\omega_{\boldsymbol{p}+\boldsymbol{q}}+q_{0}}\right)\right\}
\end{aligned}
$$

where $\omega_{\boldsymbol{p}}$ and $\Omega_{\boldsymbol{p}}$ are the energies of the $\pi$ and $\sigma$ particles with momentum $\boldsymbol{p}: \omega_{\boldsymbol{p}}=p$, $\Omega_{\boldsymbol{p}}=\left(p^{2}+m_{\sigma}^{2}\right)^{1 / 2}$; and $f(\omega)$ is the Bose-Einstein distribution function $f(\omega)=\left(e^{\beta \omega}-1\right)^{-1}$.

We need to compute the coefficients of $q_{0}^{2}$ and $q^{2}$ in the expansion of $\Sigma(Q)$. To compute the coefficient of $q^{2}$, one can set $q_{0}=0$ and Eq. (5.9) becomes

$$
\Sigma(0, \boldsymbol{q})=4 \lambda^{2} v^{2} \int \frac{d \boldsymbol{p}}{(2 \pi)^{3}} \frac{1}{\Omega_{\boldsymbol{p}}^{2}-\omega_{\boldsymbol{p}+\boldsymbol{q}}^{2}}\left[\frac{f\left(\Omega_{\boldsymbol{p}}\right)+\frac{1}{2}}{\Omega_{\boldsymbol{p}}}-\frac{f\left(\omega_{\boldsymbol{p}+\boldsymbol{q}}\right)+\frac{1}{2}}{\omega_{\boldsymbol{p}+\boldsymbol{q}}}\right] .
$$

The right hand side of Eq. (5.10) can be expanded in powers of $q$. The constant is compensated by the other diagrams. The $q^{2}$ term gives rise to an integral which is dominated in the infrared region $p \sim m_{\sigma}$, and is of order

$$
\Sigma(0, \boldsymbol{q}) \sim \frac{\lambda^{2} v^{2} T}{m_{\sigma}^{3}} \boldsymbol{q}^{2}=O\left(\lambda^{1 / 2}\right) \boldsymbol{q}^{2}+\cdots .
$$

In the last equation we assumed $T \sim v_{0}$, and $m_{\sigma}^{2} \sim \lambda T$, which is valid when $T$ is not very close to $T_{c}$. 
Now let us put $\boldsymbol{q}=0$ and expand in $q_{0}$. Since the constant term is canceled out by other diagrams, one has to look only at terms of order $q_{0}^{2}$. One finds

$$
\Sigma\left(q_{0}, \mathbf{0}\right)=-8 \lambda^{2} v^{2} q_{0}^{2} \int \frac{d \boldsymbol{p}}{(2 \pi)^{3} 2 \omega_{\boldsymbol{p}} 2 \Omega_{\boldsymbol{p}}}\left[\frac{f\left(\omega_{\boldsymbol{p}}\right)+f\left(\Omega_{\boldsymbol{p}}\right)+1}{\left(\Omega_{\boldsymbol{p}}+\omega_{\boldsymbol{p}}\right)^{3}}+\frac{f\left(\omega_{\boldsymbol{p}}\right)-f\left(\Omega_{\boldsymbol{p}}\right)}{\left(\Omega_{\boldsymbol{p}}-\omega_{\boldsymbol{p}}\right)^{3}}\right] .
$$

The first term in the square brackets gives rise to an integral that is dominated by the infrared, i.e., by $p \sim m_{\sigma}$, and so is completely analogous to the coefficient of $p^{2}$ above. The coefficient of $q_{0}^{2}$ coming from the first term is hence of order $O\left(\lambda^{1 / 2}\right)$. In contrast, the integral of the second term in the square brackets is dominated by $p \sim T$. For such $p$ one can write, approximately,

$$
\Omega_{\boldsymbol{p}}-\omega_{\boldsymbol{p}}=\frac{m_{\sigma}^{2}}{2 p}, \quad f\left(\omega_{\boldsymbol{p}}\right)-f\left(\Omega_{\boldsymbol{p}}\right)=-\frac{m_{\sigma}^{2}}{2 p} \frac{\partial n(p)}{\partial p}
$$

which gives

$$
\Sigma\left(q_{0}, \mathbf{0}\right)=\frac{8 \lambda^{2} v^{2}}{m_{\sigma}^{4}} q_{0}^{2} \int \frac{d \boldsymbol{p}}{(2 \pi)^{3}} \frac{\partial f(p)}{\partial p}=-\frac{4 \lambda^{2} v^{2}}{3 m_{\sigma}^{4}} T^{2} q_{0}^{2} .
$$

Substituting $m_{\sigma}^{2}=2 \lambda v^{2}$, we finally find

$$
\Sigma\left(q_{0}, \boldsymbol{q}\right)=-\frac{T^{2}}{3 v^{2}} q_{0}^{2} .
$$

Since $T$ and $v$ are both of order $v_{0}$, the coefficient in front of $q_{0}^{2}$ is of order $\mathcal{O}\left(\lambda^{0}\right)$, in contrast to that of $\boldsymbol{q}^{2}$. The velocity of pions in our model is

$$
u^{2}=\left(1+\frac{T^{2}}{3 v^{2}}\right)^{-1}, \quad T \gg m_{\sigma} .
$$

It is instructive to compare this result to the one obtained in the framework of the chiral perturbation theory: $u=1-\mathcal{O}\left(T^{4} / f_{\pi}^{4}\right)$ [19, 20]. One should bear in mind that the regime in which the chiral perturbation theory result applies corresponds to $T \ll m_{\sigma}$. In our linear sigma model we consider a different regime: $T \sim v \gg m_{\sigma} \sim \sqrt{\lambda} v$. In the chiral perturbation theory $T / f_{\pi}$ serves as an expansion parameter. In our weak-coupling calculation the expansion is in $\lambda$ while the $T$ dependence is included to all orders in $T / v$.

Now we need to check that this coincides with $f^{2} / \chi_{I 5}$. Recall that our definition of $f^{2}$ is as follows: if we define $\varphi^{a}=\pi^{a} / v$, then

$$
\int d \boldsymbol{x} e^{-i \boldsymbol{q} \cdot \boldsymbol{x}}\left\langle\varphi^{a}(t, \boldsymbol{x}) \varphi^{b}(t, \mathbf{0})\right\rangle=\frac{T}{f^{2}} \frac{\delta^{a b}}{\boldsymbol{q}^{2}} .
$$

The left hand side is proportional to the $\pi$ Matsubara propagator, summed over $q_{0}$,

$$
\frac{T}{v^{2}} \sum_{q_{0}} \frac{1}{q_{0}^{2}+\boldsymbol{q}^{2}} .
$$

When $|\boldsymbol{q}| \ll T$ the dominant term in the sum is the one with $q_{0}=0$, therefore,

$$
f^{2}=v^{2}
$$


Taking into account one-loop graphs, as we have seen, will change $f^{2}$ only by an amount of order $O\left(\lambda^{1 / 2}\right)$.

Now to compute the susceptibility $\chi_{I 5}$ we need to turn on a chemical potential coupled to a broken charge. There are $N-1$ broken generators. Let us consider the one that transforms $\sigma$ and $\pi_{1}$ into each other. The change of the Lagrangian when the corresponding chemical potential is turned on is

$$
\delta \mathcal{L}=\mu\left(\pi_{1} \partial_{0} \sigma-\sigma \partial_{0} \pi_{1}\right)+\frac{\mu^{2}}{2}\left[(v+\sigma)^{2}+\pi_{1}^{2}\right] .
$$

The susceptibility can be computed in Matsubara formalism. There are two contributions: one from the $\mu^{2}$ term in $\delta \mathcal{L}$, the other from the bubble graph:

$$
\begin{aligned}
\chi_{I 5} & =v^{2}+2 T \sum_{p_{0}} \int \frac{d \boldsymbol{p}}{(2 \pi)^{3}} \frac{1}{p_{0}^{2}+p^{2}}-4 T \sum_{p_{0}} \int \frac{d \boldsymbol{p}}{(2 \pi)^{3}} \frac{p_{0}^{2}}{\left(p_{0}^{2}+p^{2}\right)^{2}} \\
& =v^{2}+\frac{1}{2 T} \int \frac{d \boldsymbol{p}}{(2 \pi)^{3}} \sinh ^{-2} \frac{p}{2 T}=v^{2}+\frac{T^{2}}{3} .
\end{aligned}
$$

The interpretation of this formula is rather direct: $v^{2}$ is the contribution of the condensate, and $T^{2} / 3$ is the contribution from the free gas of $\sigma$ and $\pi_{1}$. The square of the velocity of pions, Eq. (5.16), is equal to the ratio of $f^{2}$ in Eq. (5.19) and $\chi_{I 5}$ in Eq. (5.21), which is what we need to verify. We did not, however, attempted to turn on an explicit symmetry breaking and verify, e.g., the GOR relation at finite temperature. Such a calculation should be straightforward.

\section{CONCLUSION}

Our goal has been to demonstrate that the dispersion relation of pions can be expressed in terms of quantities obtainable from equal-time or static correlation functions. The precise relation is given in Sec. 四. Our result enables one to find the real part of the pion dispersion relation on the lattice. However, it does not enable one to compute the imaginary part, which characterizes the damping of pion modes.

Nowhere in our treatment did we assume any condition on the temperature (except $T<$ $T_{c}$ ), as long as we stay infinitesimally close to the chiral limit. Thus, if $m_{q}$ is infinitesimally small, our result applies to all temperatures smaller than the temperature of the chiral phase transition $T_{c}$. However, at any fixed (small) value of $m_{q}$, our results do not apply at temperatures too close to critical, where the pion screening mass becomes of the same order as the screening mass of the sigma meson. The width of this region near $T_{c}$ shrinks to

zero as a power of $m_{q}$ (more precisely, as $m_{q}^{1 / \beta \delta}$ ). Our treatment must fail there because, as explained in Sec. पV, the sigma boson also needs to be included into the hydrodynamic theory. However, the scaling of different quantities in this temperature region can be determined 
using scaling and universality arguments, as discussed in Ref. [9]. In the Appendix of the present paper we derive some additional interesting scaling properties omitted in Ref. [9].

Finally, assuming $m_{q}$ to be very small, as $T \rightarrow T_{c}$, the chiral condensate $\langle\bar{\psi} \psi\rangle \rightarrow 0$. As shown in Ref. [9], this implies that also $f \rightarrow 0$ (although with a slightly different critical exponent). On the other hand, the axial isospin susceptibility $\chi_{I 5}$ becomes degenerate with the (vector) isospin susceptibility at $T_{c}$, where both remains finite. One concludes from Eq.

(2.2) that the pion velocity $u$ tends to zero as one approaches the critical temperature. In fact, it can be shown that $u$ approaches zero faster than the divergence of the screening mass $m$, so the pion pole mass $m_{p}=u m$ goes to zero as $T \rightarrow T_{c}$ [9].

\section{Acknowledgments}

We thank Daniel Boyanovsky, Sangyong Jeon, Guy Moore, Thomas Schäfer, Edward Shuryak and Larry Yaffe for stimulating discussions. The authors thank RIKEN, Brookhaven National Laboratory, and the U.S. Department of Energy (DE-AC0298CH10886) for providing the facilities essential for the completion of this work. D.T.S. is supported, in part, by DOE Grant No. DOE-ER-41132 and by the Alfred P. Sloan Foundation. M.A.S. is supported by a DOE OJI grant.

\section{APPENDIX A: DYNAMICAL SCALING AT THE CHIRAL TRANSITION}

In this appendix we provide a simple derivation of the dynamical critical exponent $z$ characterizing critical slowing down near the QCD chiral phase transition. It is based on a scaling argument similar to the one used in Ref. [18] to find $z$ in antiferromagnets.

For simplicity, here we consider the chiral limit $m_{q}=0$. Scaling and universality arguments presented in Ref. [9] predict that the velocity $u$ vanishes as $T$ approaches $T_{c}$ from below. The quantity $u^{2}$ scales as $u^{2} \sim t^{(d-2) \nu}$, where $t \equiv\left(T_{c}-T\right) / T_{c}$. Since the inverse correlation length of the order parameter $\bar{\psi} \psi$, i.e., the static screening mass of the sigma particle $m_{\sigma}$, scales as $m_{\sigma} \sim t^{\nu}$, we conclude that for $t \ll 1$

$$
u^{2} \sim m_{\sigma}^{d-2}
$$

The fact that $u \rightarrow 0$ at $T_{c}$ means that the dispersion relation ceases to be linear. Moreover, the effect of damping also becomes important; in other words, we expect the real and imaginary parts of $\omega$ to become comparable. In such a situation on cannot refer to $\omega$ as a quasiparticle energy. Rather, it is a characteristic frequency, or inverse relaxation time, of a mode of a given wave number $\boldsymbol{p}$. Scaling hypothesis dictates that the relation between $\boldsymbol{p}$ and $\omega$ should be homogeneous, i.e., $\omega \sim|\boldsymbol{p}|^{z}$. The dynamical scaling exponent $z$ is, in a generic case, new exponent independent of the static exponents, e.g., $\nu$ and $\eta$. However, in the case of QCD (similar to the case of an antiferromagnet [6, 18]), since the dispersion 
relation of pions is given in terms of static quantities only, it turns out that $z$, as one would expect, can be derived from static scaling laws only.

To determine $z$ we observe that the dispersion relation $\omega \sim|\boldsymbol{p}|^{z}$ applies at scales $m_{\sigma} \ll$ $|\boldsymbol{p}| \ll T$. At softer scales, $|\boldsymbol{p}| \ll m_{\sigma}$, the dispersion relation is still linear, $\omega=u|\boldsymbol{p}|$. Requiring that the two expressions for $\omega$ match at $|\boldsymbol{p}| \sim m_{\sigma}$ we find

$$
m_{\sigma}^{z} \sim u m_{\sigma} \sim m_{\sigma}^{d / 2}
$$

where to obtain the last scaling relation we used Eq. (A1). Therefore, at the QCD chiral phase transition the dynamical critical exponent is given by

$$
z=\frac{d}{2}
$$

(see also an alternative derivation in Refs. [6, 18] ). ${ }^{6}$

A similar argument can be applied to determine the scaling of the diffusion coefficient $D^{\prime}=D+\kappa_{2}$, characterizing the pion damping in the chiral limit [Eq. (4.22b)]. The small momentum expansion of $\omega$ is $u|\boldsymbol{p}|-\frac{i}{2} D^{\prime} \boldsymbol{p}^{2}+\ldots$. The scaling hypothesis dictates that all terms in this expansion become of the same order in magnitude when $|\boldsymbol{p}| \sim m_{\sigma}$ (otherwise, another scale, e.g., $u / D^{\prime}$, appears in addition to the inverse correlation length $\left.m_{\sigma}\right)$. This requires

$$
D^{\prime} \sim m_{\sigma}^{(d-4) / 2} \sim t^{\nu(d-4) / 2} .
$$

Thus $D^{\prime}$ diverges near the chiral phase transition in QCD $(d=3)$.

[1] G. Q. Li, C. M. Ko, and G. E. Brown, Phys. Rev. Lett. 75, 4007 (1995) nucl-th/9504025; Nucl. Phys. A606, 568 (1996) nucl-th/9608040; W. Cassing, W. Ehehalt, and C. M. Ko, Phys. Lett. B 363, 35 (1995) hep-ph/9508233.

[2] F. Karsch, E. Laermann, P. Petreczky, S. Stickan, and I. Wetzorke, Phys. Lett. B 530, 147 (2002) hep-lat/0110208.

[3] A. Bijl, Physica (Amsterdam) 8, 655 (1940); R. P. Feynman, in Progress in Low Temperature Physics, edited by C. J. Gorter (North-Holland, Amsterdam, 1955), Vol. 1; see also R. P. Feynman, Statistical Mechanics (Benjamin, Reading, MA, 1972)

[4] B. I. Halperin and P. C. Hohenberg, Phys. Rev. 188, 898 (1969).

[5] R. D. Pisarski and F. Wilczek, Phys. Rev. D 29, 338 (1984).

[6] K. Rajagopal and F. Wilczek, Nucl. Phys. B399, 395 (1993) hep-ph/9210253.

[7] F. C. Hansen and H. Leutwyler, Nucl. Phys. B350, 201 (1991).

[8] A. V. Smilga, Phys. Rep. 291, 1 (1997) [hep-ph/9612347.

${ }^{6}$ This result agrees with [6, 18], but disagrees with [22]. 
[9] D. T. Son and M. A. Stephanov, Phys. Rev. Lett. 88, 202302 (2002) hep-ph/0111100.

[10] J. B. Kogut, M. A. Stephanov, and D. Toublan, Phys. Lett. B 464, 183 (1999) [hepph/9906346]; J. B. Kogut, M. A. Stephanov, D. Toublan, J. J. Verbaarschot, and A. Zhitnitsky, Nucl. Phys. B582, 477 (2000) hep-ph/0001171.

[11] D. T. Son and M. A. Stephanov, Phys. Rev. Lett. 86, 592 (2001) hep-ph/0005225; Yad. Fiz. 64, 899 (2001) [Phys. At. Nucl. 64, 834 (2001)] hep-ph/0011365].

[12] S. Gottlieb, W. Liu, D. Toussaint, R. L. Renken, and R. L. Sugar, Phys. Rev. Lett. 59, 2247 (1987); S. Gottlieb, U. M. Heller, A. D. Kennedy, S. Kim, J. B. Kogut, C. Liu, R. L. Renken, D. K. Sinclair, R. L. Sugar, D. Toussaint, and K. C. Wang, Phys. Rev. D 55, 6852 (1997) hep-lat/9612020.

[13] E. V. Shuryak, Phys. Rev. D 42, 1764 (1990).

[14] J. L. Goity and H. Leutwyler, Phys. Lett. B 228, 517 (1989).

[15] L. P. Kadanoff and P. C. Martin, Ann. Phys. (N.Y.) 24, 419 (1963); D. Forster, Hydrodynamic Fluctuations, Broken Symmetry, and Correlation Functions (Benjamin, Reading, MA, 1975).

[16] L. G. Yaffe (private communication).

[17] D. T. Son, Phys. Rev. Lett. 84, 3771 (2000) hep-ph/9912267.

[18] P. C. Hohenberg and B. I. Halperin, Rev. Mod. Phys. 49, 435 (1977).

[19] R. D. Pisarski and M. Tytgat, Phys. Rev. D 54, 2989 (1996) hep-ph/9604404.

[20] D. Toublan, Phys. Rev. D 56, 5629 (1997) hep-ph/9706273.

[21] J. I. Kapusta, Finite Temperature Field Theory (Cambridge University Press, Cambridge, U.K., 1989).

[22] D. Boyanovsky and H. J. de Vega, Phys. Rev. D 65, 085038 (2002) hep-ph/0110012. 